УДК $62.868 ; 67.05 ; 66.041$

\title{
ИССЛЕДОВАНИЕ НЕЛИНЕЙНОЙ МОДЕЛИ ПОДВИЖНОЙ ПОДОВОЙ ПЛАТФОРМЫ ЭЛЕКТРИЧЕСКОЙ ПЕЧИ ДЛЯ ОБЖИГА СЫПУЧИХ МИНЕРАЛОВ
}

\author{
Нижегородов Анатолий Иванович', \\ nastromo_irkutsk@mail.ru
}

Гаврилин Алексей Николаевич², tom-gawra|@list.ru

Мойзес Борис Борисович², mbb@tpu.ru

\section{Кладиев Сергей Николаевич², kladiev@tpu.ru}

\author{
Хамитов Рустам Нуриманович ${ }^{3,4}$, \\ apple_27@mail.ru \\ 1 Иркутский национальный исследовательский технический университет, \\ Россия, 664074, г. Иркутск, ул. Лермонтова, 83. \\ 2 Национальный исследовательский Томский политехнический университет, \\ Россия, 634050, г. Томск, пр. Ленина, 30. \\ ${ }^{3}$ Омский государственный технический университет, \\ Россия, 644050, г. Омск, пр. Мира, 11. \\ ${ }^{4}$ Тюменский индустриальный университет, \\ Россия, 625000, г. Тюмень, ул. Володарского, 38.
}

Актуальность. Электрические печи с подвижной подовой платформой имеют недостатки в конструкции системы вибротранспортирования - в расположенной под углом к горизонтальной плоскости самой платформы. Актуально использование горизонтальной подовой платформы, исключающей самопроизвольное скатывание округлых частиц материала и устраняющей возможность возникновения температурной тяги внутри пространства обжига, приводящей к охлаждению нагревательной системы печи и обжигаемого сыпучего материала.

Цель работы состоит в исследовании динамики подвижной платформы с односторонним упругим элементом, обеспечивающим сильно выраженную нелинейную упругую характеристику, и процесса вибротранспортирования сыпучего материала по поверхности горизонтальной подовой плиты.

Объект: подвижная подовая платформа электрической печи для обжига сыпучих минералов.

Методика исследования базируется на разработке конструкции и модели подвижной подовой платформы электрической печи для обжига сыпучих минералов.

Результаты исследования свойств специального упругого элемента подвижной подовой платформы печи позволили получить нелинейную аналитическую модель ее движения, характеризующуюся наклонной “скелетной» кривой ее амплитудно-частотной характеристики. Исследование динамики колебательной системы с односторонним упругим элементом, обеспечивающим сильно выраженную нелинейность, подтвердило, что система совершает несимметричные колебания с пиковыми значениями виброускорения 8,32 и 4,79 м/с², соответствующими крайним положениям платформы и отличающимися в 1,74 раза. Показано, что при горизонтальной установке печи почти в три раза снижается чувствительность системы к флуктуациям частоты возбуждения, жесткости пружин, сил трения и других факторов, изменяющихся из-за влияния высокой температуры и внешних факторов. Подтвержден эффект устойчивого вибротранспортирования сыпучего материала по горизонтальной поверхности подовой плиты в тепловом поле печи.

\section{Ключевые слова:}

Электрическая печь с подвижной подовой платформой, динамическая система, нелинейный упругий элемент, “скелетная» кривая, амплитудно-частотная характеристика, чувствительность динамической системы, вибротранспортирование, не симметричные колебания.

\section{Введение}

Электрические печи с подвижной подовой платформой представляют собой энерготехнологические агрегаты для обжига и термоактивации различных минералов. Они состоят из двух систем: нагревательной - создающей регулируемое по мощности тепловое излучение, поглощаемое обрабаты- ваемым материалом, и вибротранспортной - обеспечивающей устойчивое однонаправленное и управляемое движение сыпучего материала по поверхности подовой плиты в тепловом поле.

Энергетические аспекты их функционирования изучены и рассмотрены в целом ряде работ авторов [1-8], а в работе [9] показано, что при обжи- 
ге, например, вермикулита, достигнуто значение удельной энергоемкости $75 . .80$ мДж/ $\mathrm{m}^{3}$, что в два-пять раз ниже, чем в других электрических или огневых печах обжига.

Однако механическая часть - система вибротранспортирования, имеет недостатки, которые требуют совершенствования этих печных агрегатов.

Динамическая система печи - подвижная подовая платформа, расположенная под углом к горизонтальной плоскости, характеризуется высокой чувствительностью к флуктуациям частоты возбуждения, собственной частоты, жесткости пружин, сил трения и других факторов, обусловленных действием высоких температур, запыленностью и нестабильностью электрической сети.

Так как система линейна, из-за высокой добротности, около 20 Дб, вблизи резонансной зоны амплитудно-частотной характеристики (АЧХ) незначительное изменение частоты возбуждения приводит к значительному изменению амплитуды колебаний $[10,11]$, а это в нашем случае влечет за собой изменение скорости вибротранспортирования и времени обжига.

При наклонном положении подовой платформы округлые частицы сыпучих материалов могут начать двигаться самопроизвольно, а не за счет вибротранспортного эффекта и их движение будет неконтролируемым и ускоренным, что уменьшит время термообработки таких частиц.

Кроме того, наклонное положение из-за температурной тяги будет вызывать встречное движение холодного наружного воздуха в пространстве обжи- га, где расположена нагревательная система. Очевидно, что поток воздуха будет охлаждать нагревательные элементы и движущийся материал, а это отрицательно скажется на энергоемкости обжига.

Для уменьшения чувствительности динамической системы и устранения двух других неблагоприятных факторов, необходима горизонтальная установка подвижной подовой платформы - это наиболее рациональный вариант конструкции.

Но надо создать нелинейную динамическую систему, в которой транспортный эффект возникает из-за выраженной несимметрии колебаний. А такие системы содержат нелинейные упругие элементы и имеют АЧХ с круто наклоненным влево резонансным пиком [10-13].

\section{Устройство и динамика печного агрегата с наклонной подовой платформой}

На рис. 1 показана электрическая печь с наклонной подвижной подовой платформой. Основой печи является рама - 1 . В направляющих рамы на подшипниках - 2 размещена подвижная подовая платформа - 3, подпружиненная с обеих сторон пружинами - 4. Платформа содержит металлоконструкции и термоизолирующий материал, а также подовую плиту - 5 , по которой благодаря эксцентриковому приводу -6 с плунжером - 7 и пружиной -8 движется обжигаемый сыпучий материал и выходит как готовый продукт. В пространстве обжига между термокрышкой - 9 и плитой - 5 расположена нагревательная система печи, состоящая из последовательно соединенных п-об-

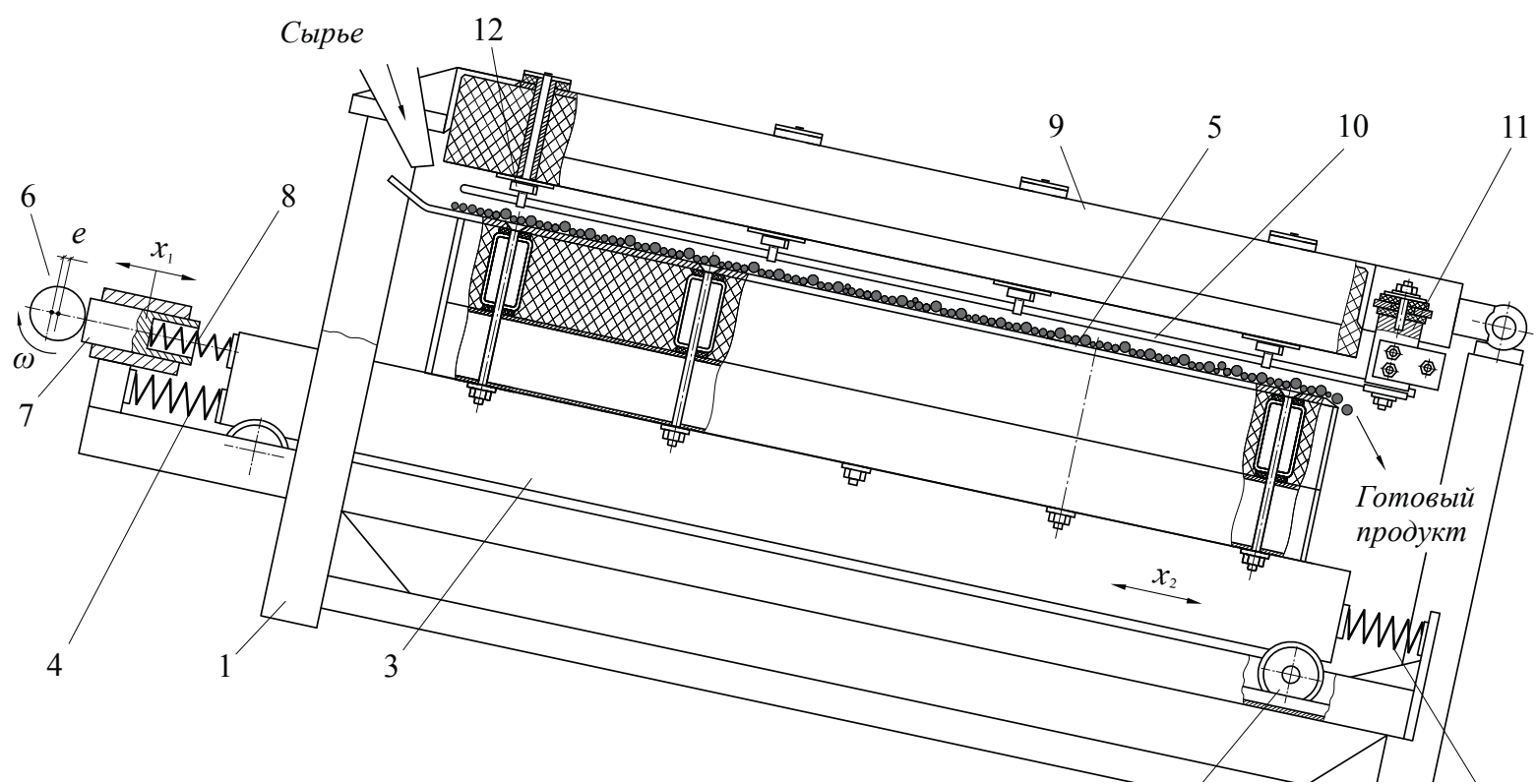

Pис. 1. Электрическая печь с наклонной подовой платформой: 1 - рала; 2 - подшипники; 3 - подвижная подовая платфорла; 4 - пружина; 5 - плита; 6 - эксцентриковый привод; 7 - плунжер; 8 - пружина; 9 - термокрышка; 10 - нагревательные элеленты; 11 - специальные головки; 12 - хомутики

Fig. 1. Electric furnace with an inclined base plate: 1 is the frame; 2 are the bearings; 3 is the mobile base plate; 4 is the spring; 5 is the plate; 6 is the eccentric drive; 7 is the plunger; 8 is the spring; 9 is the thermal cover; 10 are the heating elements; 11 are the special heads; 12 are the clamps 
разных нагревательных элементов - 10, закрепленных на специальных головках - 11 и поддерживаемых креплениями в виде хомутиков - 12 .

Не вдаваясь подробно в конструкцию нагревательной системы печи, рассмотрим работу подвижной подовой платформы - 3 , которая совместно с пружинами - 4, 8 образует динамическую систему, совершающую колебания вблизи резонансного пика.

Регулируемый частотный электропривод (на рис. 1 не показан) приводит во вращение эксцентриковый вал (e - эксцентриситет), который вызывает возвратно-поступательное движение плунжера - 7. Пружина - 8, испытывающая деформацию от двух колебаний - плунжера $x_{1}$ и платформы $x_{2}$, создает возбуждающую силу $F(\mathrm{H})$, равную:

$$
F=c_{1}\left(x_{1}-x_{2}\right) \text {. }
$$

Если не учитывать влияние присоединенных масс частиц сыпучего материала из-за их малости, то дифференциальное уравнение, описывающее динамику такой системы, имеет вид:

$$
M \frac{d^{2} x_{2}}{d t^{2}}+\alpha \frac{d x_{2}}{d t}+4 c x_{2}=c_{1}\left(x_{1}-x_{2}\right),
$$

где $\alpha$ - коэффициент сопротивления, зависящий от скорости движения, кг/с; $M$ - масса подвижной подовой платформы, кг; $c$ - жесткость пружин платформы (всего четыре штуки), кг/с $c^{2} c_{1}-ж е$ сткость пружины плунжера, кг $/ \mathrm{c}^{2} ; x_{1}$ - координата плунжера, определяемая выражением:

$$
x_{1}=e \sin \omega t,
$$

$x_{2}$ - координата платформы; $\omega$ - угловая скорость вращения эксцентрика, рад/с; $t$ - время, с. В преобразованном виде уравнение (2) принимает вид:

$$
M \frac{d^{2} x_{2}}{d t^{2}}+\alpha \frac{d x_{2}}{d t}+4 c x_{2}+c_{1} x_{2}=c_{1} x_{1} .
$$

Это система с кинематическим возбуждением, обладающая свойствами линейных систем и совершающая колебания как гармонический осциллятор [11, 14-20].

Собственная частота такой системы определяется выражением:

$$
\omega_{0}=\sqrt{\frac{4 c+c_{1}}{M}},
$$

а коэффициент демпфирования колебаний, определяющий добротность системы, по формуле:

$$
\xi=\frac{\alpha}{2 \sqrt{m\left(4 c+c_{1}\right)}} .
$$

Для динамических систем на телах качения $\xi$ примерно равен $0,07 . . .0,075$, поэтому система обладает высокой добротностью, когда усиление колебаний в резонансе достигает 20...21 Дб [11].

При испытаниях опытного образца печи с подвижной подовой платформой, показанной на рис. 2 (нагревательная система и термокрышка сняты), экспериментально были исследованы колебания на разных частотах возбуждения $f$ (Гц) и построена АЧХ, хорошо соответствующая линейной системе. Коэффициент динамичности составил $10 . . .11$, что соответствует усилению колебаний в резонансе 20 Дб.

Оценка коэффициента чувствительности амплитуды $A$ к изменению частоты возбуждения, определяемого отношением $\varepsilon=\Delta A / \Delta f$, показала, что вблизи резонанса система становится максимально чувствительной, а на пологом участке правой ветви АЧХ чувствительность снижается, стабильность колебаний платформы увеличивается. Следовательно, динамическую систему нужно привести к нелинейной и обеспечить наклонный резонансный пик, свойственный системам с мягкой упругой характеристикой [13].

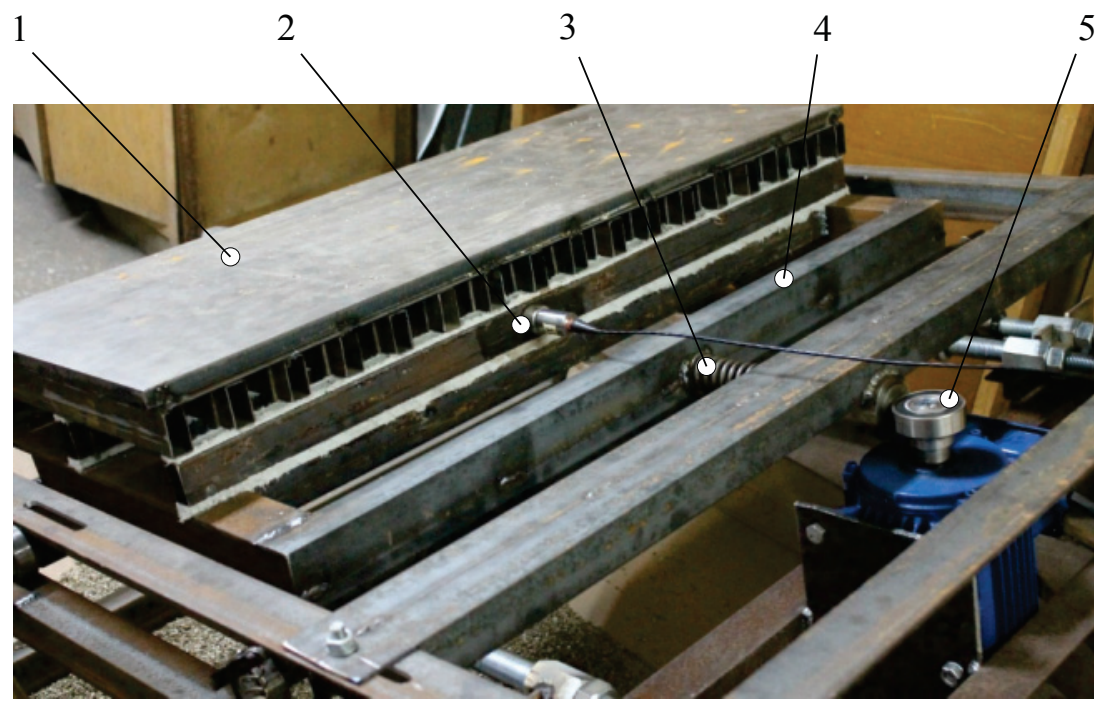

Рис. 2. Опытный образеи печи в процессе испытаний: 1 - подовая плита; 2 - вибропреобразователь; 3 - пружина толкателя; 4 - подвижная платформа; 5 - эксиентрик

Fig. 2. Prototype of the furnace during the test: 1 is the base plate; 2 is the vibration transducer; 3 is the pusher spring; 4 is the mobile base plate; 5 is the eccentric gear 


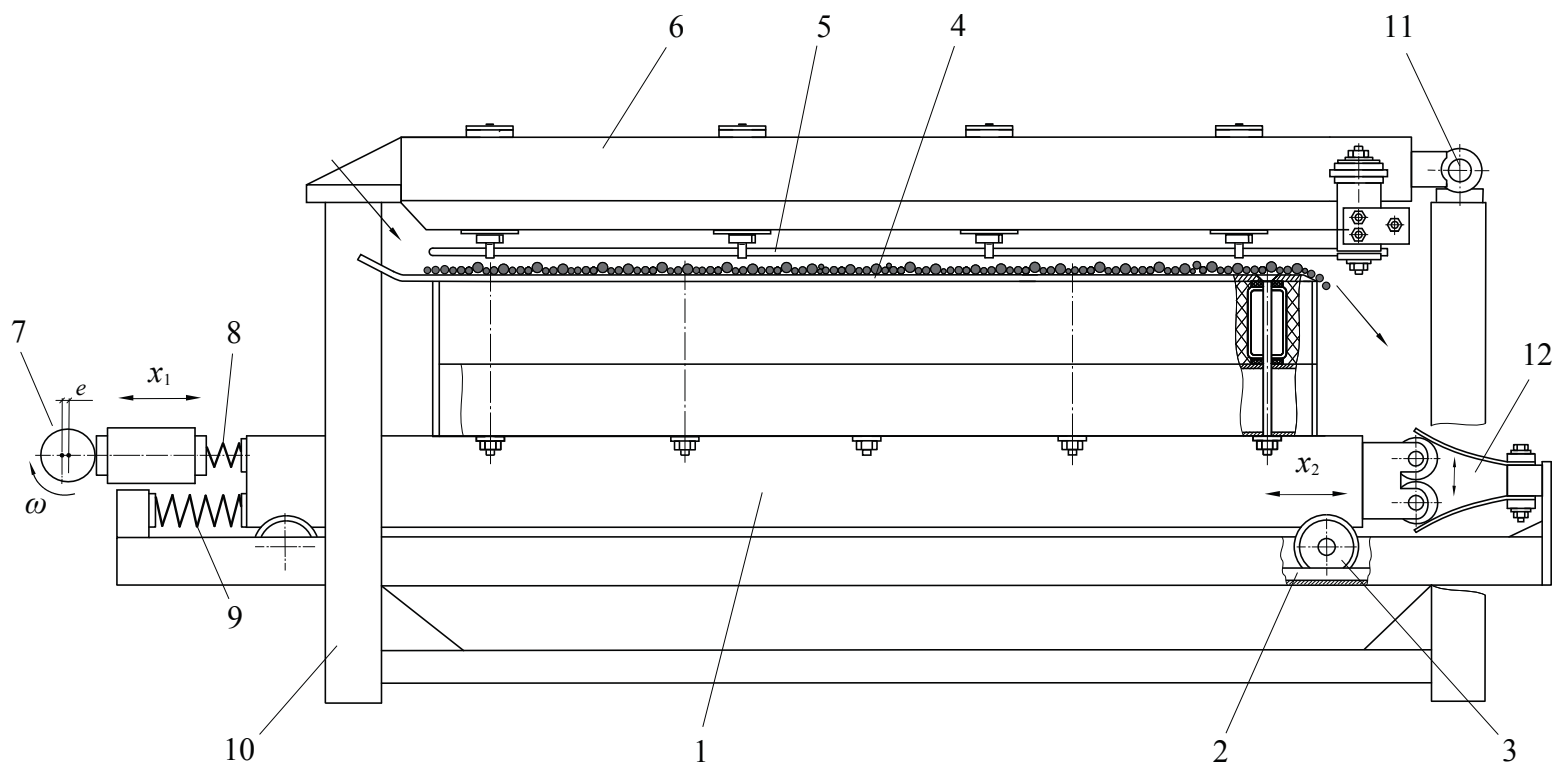

Рис.3. Электрическая печь с горизонтальной подовой платфорлой: 1 - платфорла; 2 - направляющие; 3 - подшипники; 4 - подовая плита; 5 - электронагреватели; 6- термокрышка; 7 - эксиентриковый привод; 8, 9- пружины; 10 - рала; 11 - шарнир; 12 нелинейный упругий элелент

Fig. 3. Electric furnace with a horizontal hearth platform: 1 is the platform; 2 are the guides; 3 are the bearings; 4 is the base plate; 5 are the electric heaters; 6 is the thermal cover; 7 is the eccentric drive; 8, 9 are the springs; 10 is the frame; 11 is the joint-hinge; 12 is the nonlinear elastic element

\section{Подвижная подовая платформа} с нелинейным упругим элементом

На рис. 3 показана та же печь, но с горизонтальным расположением подовой платформы - 1 , размещенной в направляющих -2 на подшипниках - 3. Печь содержит подовую плиту -4 , электронагреватели - 5, термокрышку - 6 и эксцентриковый привод -7 , включающий эксцентрик и плунжер с пружиной - 8. Пружины - 9 установлены только с левой стороны платформы. Термокрышка установлена на раме - 10 на шарнире 11 с возможностью ее открывания для доступа к электронагревателям.

C правой стороны платформы закреплен нелинейный упругий элемент - 12 с сильно выраженной зависимостью силы упругости от перемещения $x_{2}$ подвижной подовой платформы.

Конструкция упругого элемента показана на рис. 4 . Он содержит корпус -1 , закрепляемый на подвижной подовой платформе, с установленными в нем на осях -2 роликами - 3 радиуса $R$ (диаметра $D$ ), находящимися в контакте с упругими пластинами -4 толщиной $a$, выполненными из пружинной стали 55 ХГР. Указанные пластины закреплены резьбовым соединением -5 на упоре -6 , который крепится к раме печи (рис. 3).

В отличие от наклонной печи в резонансном режиме подвижная платформа, благодаря нелинейному упругому элементу, совершает асимметричные колебания. При движении платформы вправо, ролики - 2 (рис. 4), прокатываясь по упругим пластинам -4 , отгибают их в стороны, что приводит к непропорциональному увеличению силы упруго- сти $F_{\text {уп }}$ при смещении платформы на величину $x_{2}$. При этом сила упругости равна:

$$
F_{\text {yI }}=2 F_{x},
$$

где $F_{x}, F_{y}$ - горизонтальная и вертикальная составляющие равнодействующей силы $F$ в точках контакта роликов и пластин.

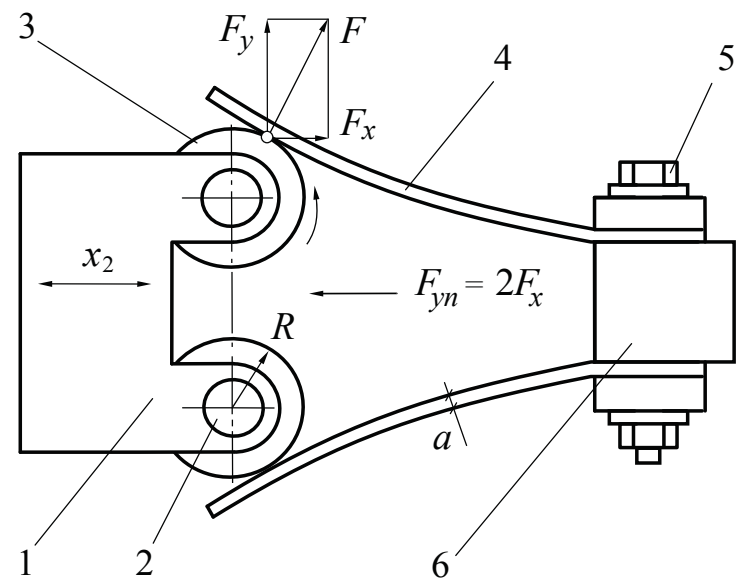

Pис. 4. Нелинейный упругий элемент: 1 - корпус; 2 - оси; 3 - ролики; 4 - упругие пластины; 5 - резьбовое соединение; 6 ynop

Fig. 4. Non-linear elastic element: 1 is the body; 2 are the axes; 3 are the rollers; 4 are the elastic plates; 5 is the threaded joint; 6 is the retainer plate

Из-за сильно выраженной нелинейной зависимости силы упругости $F_{\text {уп }}$ от перемещения $x_{2}$ подовой платформы формируется переменная жесткость упругого элемента, возрастающая по мере 
увеличения $x_{2}$ при ее движении вправо. Известно, что любая колебательная система, имеющая упругий элемент с такой зависимостью, расположенный с одной стороны, обладает «мягкой» амплитудно-частотной характеристикой [13].

Асимметричные колебания создают односторонний вибротранспортный эффект. Он характеризуется тем, что максимальное ускорение частицы, находящейся на поверхности подовой плиты, направленное вправо, не равно максимальному абсолютному ускорению частицы, направленному влево. Если максимум ускорения вправо $\ddot{x}_{2 \max }$ больше (как в данном случае), чем максимум ускорения влево $\ddot{x}_{2 \text { maxл, }}$, то выполняется условие [14]:

$$
\ddot{x}_{2 \max \Pi}>\frac{f G}{m}>\ddot{x}_{2 \max \Omega},
$$

где $f$ - коэффициент трения частицы по металлической поверхности; $m$ - масса частицы, кг; $G$ - вес частицы, Н. Частица и весь поток сыпучего материала будет двигаться вправо без отрыва от поверхности, при этом движение будет иметь однонаправленный пульсирующий характер.

\section{Исследование нелинейного упругого элемента}

Для установления зависимости между силой упругости $F_{\text {уп }}$, действующей со стороны упругого элемента, и перемещением платформы $x_{2}$, рассмотрим расчетную схему, приведенную на рис. 5 .

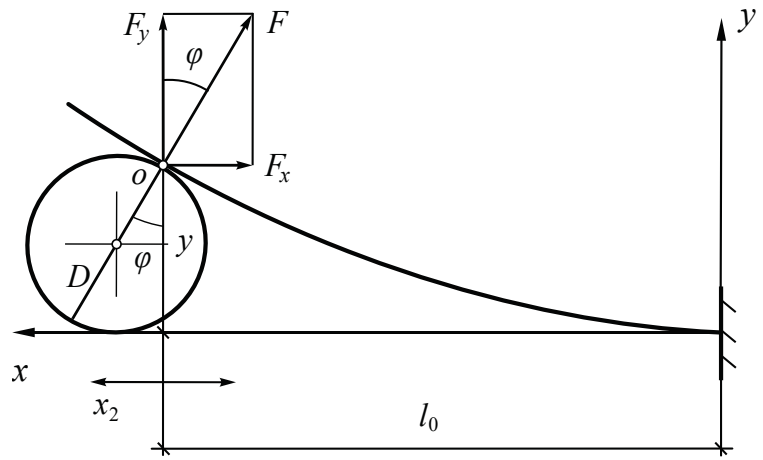

Pис. 5. Расчетнал схема упругого элемента

Fig. 5. Design scheme of the elastic element

При накатывании ролика на пластину возникает сила $F$, изгибающая ее на величину $y$. Из теории упругости известно [21-23], что искривленная ось балки, а в данном случае пластины, называется упругой линией, уравнение которой для точки 0 имеет вид (м):

$$
y=\frac{F_{y} l_{0}^{3}}{3 E J_{z}},
$$

а угол поворота $\varphi$ (рад) сечения пластины в точке 0 определяется выражением:

$$
\varphi=\frac{F_{y} l_{0}^{2}}{2 E J_{z}},
$$

где $F_{y}$ - вертикальная составляющая силы $F ; E$ - модуль упругости стали, $\mathrm{H} / \mathrm{M}^{2} ; J_{z}$ - осевой момент инер- ции поперечного сечения относительно оси $z, \mathrm{M}^{4}$.

Из уравнения (7) выразим силу $F_{y}$ :

$$
F_{y}=\frac{3 y E J_{z}}{l_{0}^{3}} .
$$

Конструкция упругого элемента выполнена так, что прогиб $у$ в исходном положении примерно равен диаметру ролика $D$, а начальная координата по оси $x$ равна $l_{0}$. Величина $y$ является переменной, но она изменяется в процессе колебаний подовой платформы незначительно. Поэтому можно принять

$$
y \approx 0,9 D=\text { const. }
$$

Тогда формула (9) примет вид:

$$
F_{y}=\frac{3 \cdot 0,9 D E J_{z}}{l_{0}^{3}}=\frac{2,7 D E J_{z}}{l_{0}^{3}} .
$$

Исходя из расчетной схемы (рис. 5), выразим горизонтальную составляющую равнодействующей $F$ :

$$
F_{x}=F_{y} \cdot \operatorname{tg} \varphi .
$$

Подставив выражения (7) и (8) в формулу (11), после преобразований с учетом формулы (10) получим:

$$
F_{x}=\frac{2,7 D E J_{z}}{l_{0}^{3}} \operatorname{tg}\left(\frac{1,35 D}{l_{0}}\right) .
$$

Для небольших углов тангенс угла равен самому углу, выраженному в радианах. Даже для угла $30^{\circ}$ расхождение значений составляет всего $10 \%$. Сделаем преобразование с учетом перевода угла в радианы и получим:

$$
F_{x}=\frac{2,7 D E J_{z}}{l_{0}^{3}}\left(\frac{180^{\circ}}{\pi} \frac{1,35 D}{l_{0}} \frac{\pi}{180^{\circ}}\right)=\frac{3,6 D^{2} E J_{z}}{l_{0}^{4}} .
$$

После преобразований получим окончательное выражение для исходного состояния рассматриваемой динамической системы:

$$
F_{x}=\frac{3,6 D^{2} E J_{z}}{l_{0}^{4}},
$$

расчетная схема которой показана на рис. 6 .

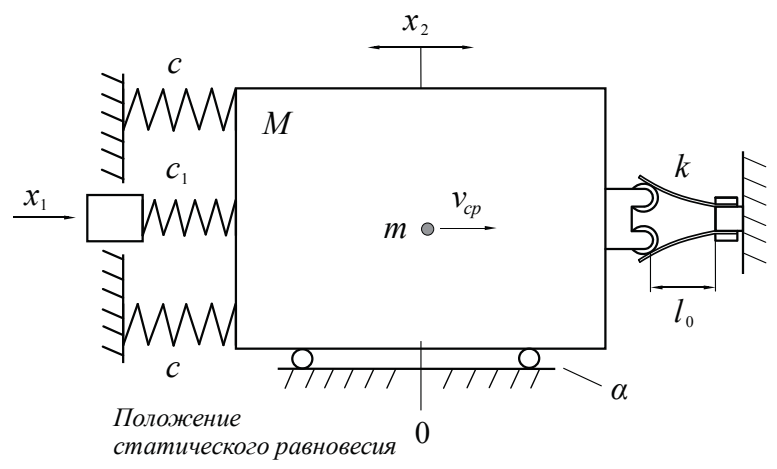

Pис. 6. Расчетная схема динамической системы

Fig. 6. Design scheme of a dynamic system

Введем обозначение числителя полученной дроби (13) 


$$
k=3,6 D^{2} E J_{z} \text {. }
$$
$\mathrm{H} \cdot \mathrm{M}^{4}$.

Размерность полученного коэффициента -

При этом, с учетом сделанных допущений, $k=$ const. Тогда формулу (13) с учетом того, что в упругом элементе находится две пластины, можно записать в виде:

$$
F_{x}=2 \frac{k}{l_{0}^{4}} .
$$

При фиксированном положении эксцентрика 7 (рис. 3) $x_{1}=0$, подовая платформа находится в положении статического равновесия: $x_{2}=0$ (рис. 6). Тогда имеет место равенство сил упругости слева $c x_{02}+c_{1} x_{02}$ и сил упругости справа $12 k / l_{0}^{4}(15)$ :

$$
2 c x_{02}+c_{1} x_{02}=2 \frac{k}{l_{0}^{4}} .
$$

Относительно этого состояния будут происходить колебания платформы:

$$
l_{0} \pm x_{2} \text { и } x_{02} \pm x_{2} \text {. }
$$

Для построения статической упругой характеристики рассматриваемой системы зададим диаметр ролика $-D=0,016$ м и начальную координату по оси $x-l_{0}=0,065 \mathrm{~m}$, исходя из пропорций на рис. 5. Чтобы рассчитать значение коэффициента $k$, зададим толщину $a=0,004$ м и ширину $в=0,03$ м пластин упругого элемента (рис. 4).

Определим осевой момент инерции поперечного сечения [22, 23]:

$$
J_{z}=\frac{a^{3} b}{12}=0,16 \cdot 10^{-9} \mathrm{~m}^{4} .
$$

Расчет по формуле (14) для пружинной стали марки 55 ХГР $\left(E=212109 \mathrm{H} / \mathrm{M}^{2}[24]\right)$ дает значение $k=0,0313 \mathrm{H} \cdot \mathrm{m}^{4}$.

При испытаниях опытного образца печи (рис. 2) предварительно экспериментально определялись масса подовой платформы $M-76,4$ кг и жесткость пружин $-c_{1}=15030 \mathrm{H} /$ м и $c=39876 \mathrm{H} / \mathrm{m}$.

Исходя из выражения (16), найдем начальную деформацию пружин $x_{02}(\mathrm{M})$, соответствующую значению $l_{0}$ при нейтральном положении эксцентрика $\left(\omega t=0\right.$ и $\left.x_{1}=0\right)$ :

$$
x_{02}=\frac{2 k}{l_{0}^{4}\left(2 c+c_{1}\right)}=\frac{2 \cdot 0,0313}{0,065^{4}(2 \cdot 39876+15030)}=0,037 \text {. }
$$

При такой начальной деформации $(0,037 \mathrm{~m})$ свободная длина пружин должна быть не менее 80 мM.

\section{Динамика горизонтальной подовой платформы}

Для получения суммарной упругой характеристики платформы относительно положения статического равновесия (рис. 6) нужно задать ряд значений $x_{2}$, смещая платформу сначала вправо $\left(-x_{2}\right.$ и $\left.l_{0}-x_{2}\right)$, а затем влево $\left(+x_{2}\right.$ и $\left.l_{0}+x_{2}\right)$, и вычесть начальную силу упругости (правая скобка) при $x_{02}=0,037 \mathrm{M}$ :

$$
\begin{aligned}
F_{\text {уп }}=\left[2 c\left(x_{02}-x_{2}\right)+c_{1}\left(x_{02}-x_{2}\right)+4 \frac{k}{\left(l_{0}-x_{2}\right)^{4}}\right]- \\
-\left[2 c_{2} x_{02}+c_{1} x_{02}+4 \frac{k}{l_{0}^{4}}\right] .
\end{aligned}
$$

Например, при значениях $x_{2}=0,002$ м и $x_{02}=0,037 \mathrm{~m}$ расчет по формуле (17) даст результат $3110+586+3107-7013=-211 \mathrm{H}$.

На рис. 7 показан график суммарной статической характеристики динамической системы (точками обозначены выборочные расчетные значения $\left.F_{\text {уп }}\right)$. Из графика видно, что система обладает нелинейной упругой характеристикой, асимметричной относительно вертикали, проходящей через начало координат.

На рис. 7 приведены четыре расчетных значения переменной жесткости $c_{x}(\mathrm{H} / \mathrm{M})$, определенные по отношению:

$$
C_{x}=\frac{F_{\mathrm{yI}}}{x_{2}} .
$$

Располагая этими значениями, определим соответствующие «мгновенные» численные значения собственной частоты, используя формулу (5), которая теперь будет иметь вид:

$$
\omega_{0}^{\prime}=\sqrt{\frac{C_{\tilde{o}}}{M}}
$$

где $M$ - масса подвижной подовой платформы, кг.

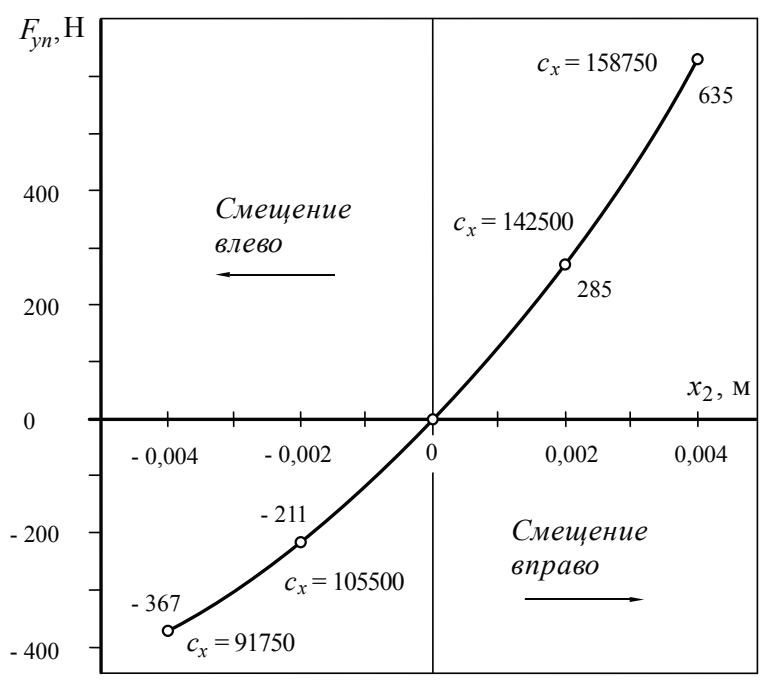

Puс. 7. Статическая упругая характеристика системы

Fig. 7. Static elastic characteristic of the system

Произведем расчеты, поставив полученным частотам в соответствие величину смещения $x_{2}$ :

$$
\begin{aligned}
& x_{2}=-0,004 \mathrm{M} \rightarrow \omega_{01}^{\prime}=34,6 \mathrm{paд} / \mathrm{c}=5,51 \text { Гц; } \\
& x_{2}=-0,002 \mathrm{M} \rightarrow \omega_{02}^{\prime}=37,2 \mathrm{paz} / \mathrm{c}=5,92 \text { Гц; } \\
& x_{2}=+0,002 \mathrm{M} \rightarrow \omega_{03}^{\prime}=43,2 \mathrm{paд} / \mathrm{c}=6,88 \text { Гц; } \\
& x_{2}=+0,004 \mathrm{M} \rightarrow \omega_{04}^{\prime}=45,6 \text { рад/c=7,26 Гц. }
\end{aligned}
$$


Установим зависимость мгновенных собственных частот от координаты платформы $x_{2}$. Левая шкала на рис. 8 соответствует искомой зависимости (график 1) и называется «скелетной» кривой [24-26].

Для расчетной схемы (рис. 6) дифференциальное уравнение (4) для наклонной подовой платформы при наличии в системе нелинейного упругого элемента 12 с двумя пластинами (рис. 3) и горизонтальном положении примет следующий вид:

$$
\begin{gathered}
M \frac{d^{2} x_{2}}{d t^{2}}+\alpha \frac{d x_{2}}{d t}+ \\
+\left[2 c\left(x_{02}-x_{2}\right)+c_{1}\left(x_{02}-x_{2}\right)+2 \frac{k}{\left(l_{0}-x_{2}\right)^{4}}\right]- \\
-\left[2 c_{2} x_{02}+c_{1} x_{02}+2 \frac{k}{l_{0}^{4}}\right]=c_{1} x_{1} .
\end{gathered}
$$

Полученное уравнение не имеет аналитического решения, поэтому построить АЧХ рассматриваемой динамической системы не удастся, но это не исключает возможности ее дальнейшего анализа.

Располагая данными о подобных системах с асимметричными упругими связями $[11,13,14,27]$ и результатами собственных экспериментальных и теоретических исследований [28], можно утверждать, что их АЧХ определяются положением «скелетных» кривых. Так, в работе [28] теоретически установлена связь скелетной кривой и АЧХ. Но в данном случае уравнение (19) не позволяет определить эту связь аналитическим путем, поэто- му можно лишь приближенно наметить линии АЧХ и оценить чувствительность системы.

На рис. 8 показаны кривые 2 и 3 - это правая и левая ветви АЧХ, построенные относительно «скелетной» кривой. Так как амплитуда $(A)$ и размах колебаний $(R=2 A)$ не могут принимать отрицательных значений, правая шкала развернута от 0 до $0,008 \mathrm{~m}$.

Здесь интерес представляет только правая (зарезонансная) ветвь. В зоне 1 вблизи резонанса коэффициент чувствительности $\varepsilon$ по отношению $\Delta A / \Delta f$ равен 1,05 мм/Гц. Для наклонной подовой платформы (рис. 1), движение которой описывается линейным дифференциальным уравнением (4), значение $\varepsilon$ равно $3,7 \mathrm{~mm} /$ ц [28], а это в 3,5 раза меньше.

В зоне 2 чувствительность становится еще меньше, так как $\varepsilon=0,37 \mathrm{мm} /$ Г.

Несмотря на то, что данные результаты можно рассматривать как приближенные, ошибка не велика. Если взять эти отношения $\Delta A / \Delta f$ на «скелетной» кривой, то они будут отличаться на $10 . . .15 \%$.

Вернемся к дифференциальному уравнению (19), описывающему динамику подовой платформы при ее горизонтальном положении. Пренебрегая трением в системе

$$
\alpha \frac{d \dot{x}_{2}}{d t}=0
$$

и приравнивая его правую часть к нулю

$$
c_{1} x_{1}=0
$$

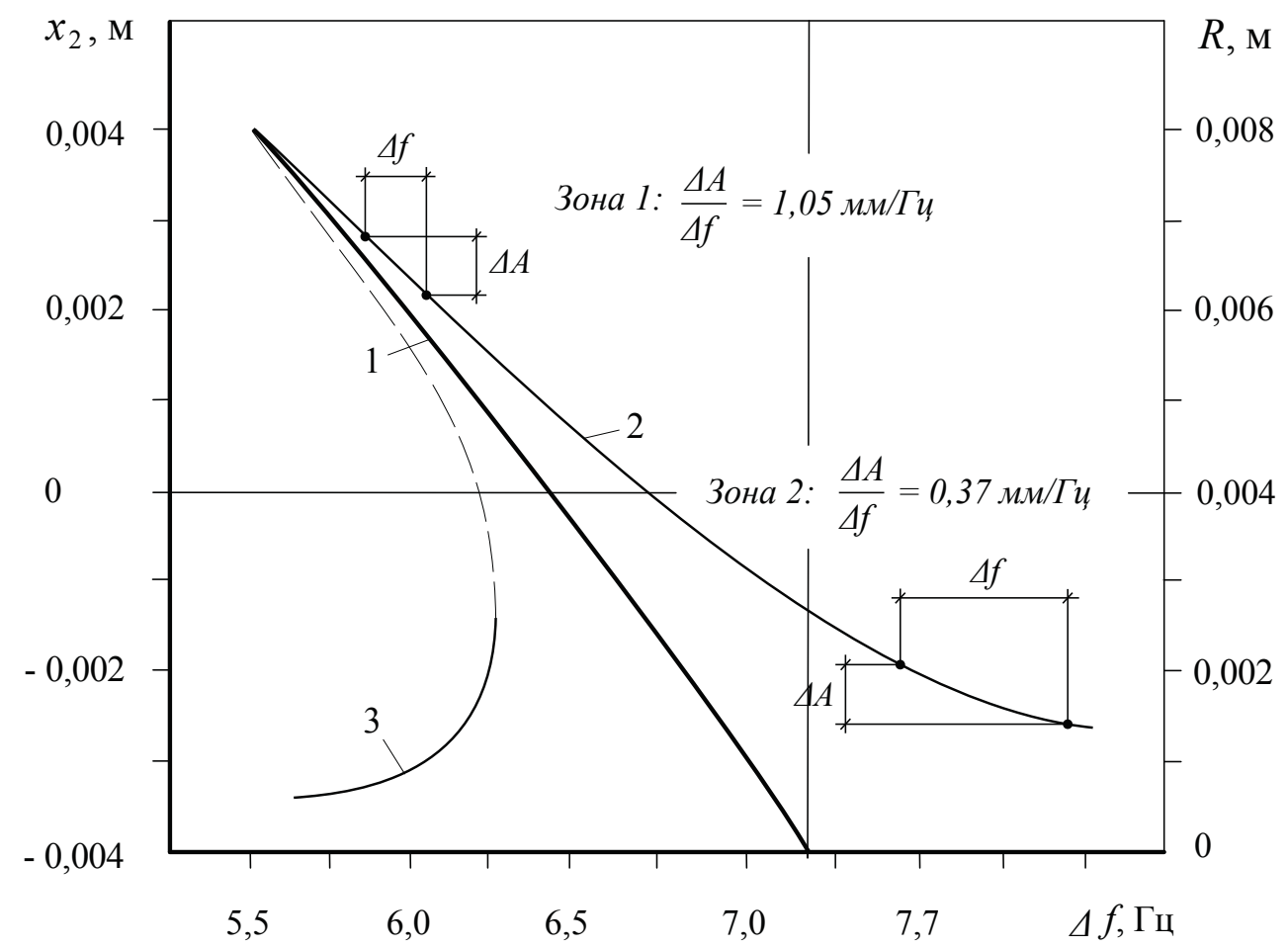

Рис. 8. «Скелетная» кривая (1) и примерные правая (2) и левая (3) ветви АЧХ

Fig. 8. «Skeletal» curve (1) and approximate right (2) and left (3) amplitude frequency response branches 
получим уравнение собственных колебаний подвижной подовой платформы:

$$
\begin{gathered}
M \frac{d^{2} x_{2}}{d t^{2}}+\left[2 c\left(x_{02}-x_{2}\right)+c_{1}\left(x_{02}-x_{2}\right)+2 \frac{k}{\left(l_{0}-x_{2}\right)^{4}}\right]- \\
-\left[2 c_{2} x_{02}+c_{1} x_{02}+2 \frac{k}{l_{0}^{4}}\right]=0 .
\end{gathered}
$$

Это уравнение консервативной динамической системы, когда из-за отсутствия возбуждающей силы в систему не подводится энергия извне, но и не рассеивается, так как трением пренебрегли. В таком случае платформа будет колебаться в незатухающем режиме при размахе $R$, равном удвоенной величине $x_{2}$, на которую мы отклоним платформу и отпустим. При этом будет идти постоянный переход кинетической энергии $T$ движущейся массы $M$ в потенциальную энергию П упругих связей: $T=\Pi=$ const, поэтому формула для ускорений записывается в виде

$$
\ddot{x}_{2 \max \Pi}=A\left(\omega_{0}^{\prime}\right)^{2} .
$$

Тогда, задавая, например, $A=+0,004$ м, получим пиковое значение виброускорения (м/ $\left.\mathrm{c}^{2}\right)$ в крайнем правом положении платформы:

$$
\ddot{x}_{2 \max \Pi}=A\left(\omega_{04}^{\prime}\right)^{2}=0,004 \cdot 45,6^{2}=8,32 .
$$

При $A=-0,004$ м получим пиковое значение виброускорения в крайнем левом положении платформы, $\mathrm{M} / \mathrm{c}^{2}$ :

$$
\ddot{x}_{2 \max \Omega}=A\left(\omega_{01}^{\prime}\right)^{2}=0,004 \cdot 34,6^{2}=4,79 .
$$

В крайнем правом положении платформы максимальное виброускорение в 1,74 раза больше, чем в крайнем левом, что указывает на выраженную асимметрию колебаний платформы $x_{2}$ и соответствует условию (6).
Точно такой же результат будет и при вынужденных колебаниях, но при условии, что уровни подводимой энергии и диссипативной (рассеиваемой трением) будут равны:

$$
E_{\text {под }}=E_{\text {дис }}
$$

и таковы, что в резонансе или вблизи резонансного пика будет обеспечена амплитуда $A= \pm 0,004 \mathrm{м}$.

На рис. 9 показаны графики асимметричных колебаний подовой платформы печи. Первый график соответствует колебаниям с амплитудой $A=0,004$ м и пиковыми значениями виброускорения 8,32 и $4,79 \mathrm{~m} / \mathrm{c}^{2}$; второй - колебаниям с амплитудой 0,002 м и пиковыми значениями виброускорения 3,7 и $2,8 \mathrm{~m} / \mathrm{c}^{2}$. Отношение виброускорений во втором режиме с уменьшенной амплитудой $(3,7 / 2,8=1,32)$ значительно меньше. Следовательно, чем сильнее проявляется асимметрия колебаний, тем больше отношение $\ddot{x}_{2 \max \Pi} / \ddot{x}_{2 \max .}$. Усиления асимметрии можно добиться увеличением размаха колебаний или применением упругих элементов с большей нелинейностью упругой характеристикой.

\section{Оценка вибротранспортного эффекта}

На примере вспученного вермикулита проверим, будет ли проявляться вибротранспортный эффект, при котором данный сыпучий материал начнет одностороннее поступательное движение по подовой плите платформы в печи обжига. Масса $m$ одной вспученной частицы с условным диаметром 0,002 м составляет $3,33 \cdot 10^{-9} \mathrm{\kappa}$ [29], а ее вес $G=3,33 \cdot 10^{-9} \cdot 9,81 \mathrm{H}$.

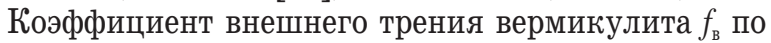
стальной поверхности равен 0,58 [30].

Из неравенства (6) определим отношение для частицы вермикулита:

$$
\frac{f_{\mathrm{B}} G}{m}>=5,69 \mathrm{M} / \mathrm{c}^{2} .
$$

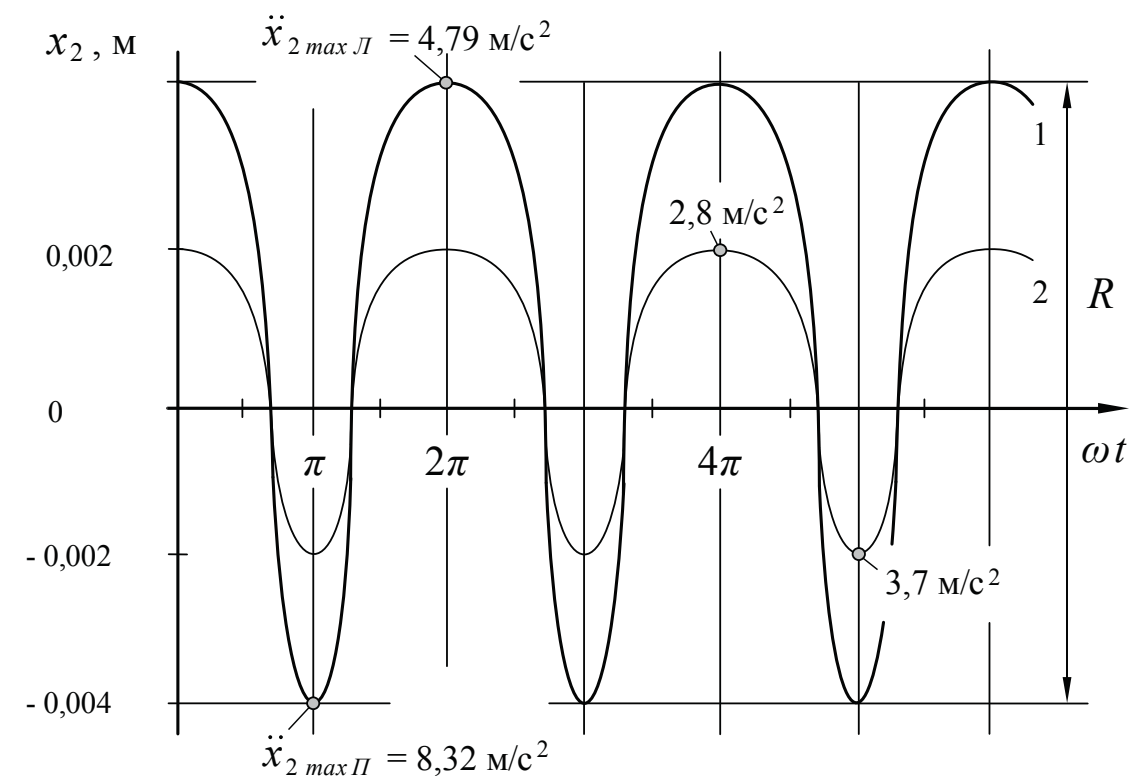

Pис. 9. Асиллетричные колебания подовой платфорлы

Fig. 9. Non-symmetrical vibrations of the base plate 
Сопоставляя полученное значение с пиковыми значениями виброускорения при амплитуде колебаний подвижной подовой платформы $A=0,004 \mathrm{M}$, получим:

$$
8,32>\frac{f_{\mathrm{B}} G}{m}>4,79 .
$$

Таким образом, условие (6) выполняется и обжигаемый в электрической печи вермикулит будет двигаться по поверхности подовой плиты поступательно с постоянной средней скоростью $v_{\text {ср }}$ (рис. 6).

Определение коэффициентов трения в работе [30] проводилось при температуре окружающего воздуха $\sim 20{ }^{\circ} \mathrm{C}$. Предполагая, что нагрев подовой плиты может повлиять на значение коэффициента $f_{в}$, возникает необходимость управлять параметрами вибрации, чтобы влиять на пиковые значения виброускорения. Это возможно за счет изменения возбуждающей силы $F(1)$ через изменение величины эксцентриситета (3). Кроме того, несложно управлять частотой возбуждения $\omega$, собственной частотой $\omega_{0}^{\prime}(18)$ и, следовательно, амплитудой $A$, изменяя начальное положение подовой платформы, оказывая влияние на параметр $l_{0}$ и суммарную упругую характеристику в целом (рис. 5-7).

\section{Заключение}

В результате исследования свойств специального упругого элемента получена аналитическая модель подвижной подовой платформы печи обжига с сильно выраженной нелинейной упругой характеристикой. Исследование динамики этой ко-

\section{СПИСОК ЛИТЕРАТУРЫ}

1. Effect of the temperature on the structural and textural properties of a compressed K-vermiculite / F. Balima, Laurence R. L., AnNgoc N., Le Floch S. // Chemical Engineering Science. - 2015. V. $134 .-$ P. 555-562.

2. Preparation and characterization of the eco-friendly chitosan/vermiculite biocomposite with excellent removal capacity for cadmium and lead / L. Chen, W.P. Pingxiao, M. Chen, T. Liu // Applied Clay Science. - 2018. - V. 159. - P. 74-82.

3. Industrial minerals \& rocks: commodities, markets, and used Chief editor J.E. Kogal. - Littleton: Society for Mining. Metallurgy, and Exploration, Inc., 2006. - 1529 p.

4. Karatas M., Benli A., Toprak H.A. Effect of incorporation of raw vermiculite as partial sand replacement on the properties of selfcompacting mortars at elevated temperature // Construction and Building Materials. - 2019. - V. 221. - P. 163-176.

5. Kariya J., Ryu J., Kato Y. Development of thermal storage material using vermiculite and calcium hydroxide // Applied Thermal Engineering. - 2016. - V. 94. - P. 186-192.

6. Ngayakamo B., Eugene Park S. Evaluation of kalalani vermiculite for production of high strength porcelain insulators // Science of Sintering. - 2019. - V. 51. - № 2. - P. 1-10.

7. Rashad A.M. Vermiculite as a construction material - a short guide for Civil Engineer // Construction and Building Materials. 2016. - V. 125 . - P. 53-62

8. Zhang Y.N. et al. Development and thermochemical characterizations of vermiculite $\mathrm{SrBr}_{2}$ composite sorbents for low-temperature heat storage // Energy. - 2016. - V. 115. - P. 120-128. лебательной системы с односторонним упругим элементом, обеспечивающим сильно выраженную нелинейность, показало, что система совершает несимметричные колебания с предельными значениями виброускорения 8,3279 и $4,79 \mathrm{M} / \mathrm{c}^{2}$, соответствующими крайним положениям платформы и отличающимися в 1,74 раза.

Проверка условия (6), при котором обеспечивается эффект вибротранспортирования сыпучего материала, подтвердила возможность его движения (на примере вермикулита) по горизонтальной подовой плите рассматриваемой печи обжига.

Как отмечалось во введении, необходимость горизонтальной установки печи была обусловлена требованием снижения чувствительности системы к флуктуациям частоты возбуждения, собственной частоты, жесткости пружин, сил трения и других факторов, обусловленных работой нагревательной системы, действием высоких температур, запыленностью, а также нестабильностью электрической сети. Эта задача решена: коэффициент чувствительности снижен более чем в три раза, в зависимости от положения рабочей точки системы на амплитудно-частотной характеристике.

Использование горизонтальной подовой платформы с нелинейным упругим элементом позволяет исключить самопроизвольное скатывание округлых частиц материала, что могло иметь место в печах с наклонным подом, а также устраняет возможность возникновения температурной тяги внутри пространства обжига под термокрышкой и охлаждения нагревательной системы печи и самого обжигаемого сыпучего материала.

9. Испытания новой альтернативной электрической печи для обжига вермикулитовых концентратов / А.И. Нижегородов, Т.Б. Брянских, А.Н. Гаврилин, Б.Б. Мойзес, А.В. Градобоев, Г.В. Вавилова // Известия Томского политехнического университета. Инжиниринг георесурсов. - 2018. - Т. 329. - № 4. C. $142-153$.

10. Slavova Y., Marinova M. On determining the energy efficiency of the electric oven $/ / 16^{\text {th }}$ Conference on Electrical Machines, Drives and Power Systems, ELMA. - Varna, Bulgaria, 2019. - Hoмер статьи 8771681.

11. Бауман В.А., Быховский И.И. Вибрационные машины и процессы в строительстве. - М.: Высш. школа, 1977. - 255 с.

12. Ahirrao N.S., Bhosle S.P., Nehete D.V. Dynamics and Vibration Measurements in Engines // Procedia Manufacturing. - 2018. V. 20. - P. 434-439.

13. Вибрации в технике: Справочник в 6-ти т. Т. 2. Колебания нелинейных механических систем / под ред. И.И. Блехмана. М.: Машиностроение, 1979. - 351 с.

14. Ден Гартог Дж. П. Механические колебания. - М.: Физматгиз, 1960. -455 c.

15. Deng N., Ostadan F. Random vibration theory based seismic site response analysis // 14 $4^{\text {th }}$ World Conference on Earthquake Engineering. - Beijing, 2008. - Номер статьи 04-02-0024.

16. Резонансные колебания с предельной амплитудой в вибрационом электромагнитном активаторе / А.Н. Гаврилин, Е.В. Боловин, А.С. Глазырин, С.Н. Кладиев, В.И. Полищук // Известия Томского политехнического университета. Инжиниринг георесурсов. - 2019. - Т. 330. - № 1. - С. 201-213. 
17. Идентификация параметров механической системы вибрационного электромагнитного активатора по граничным околорезонансным частотам / А.Н. Гаврилин, С.Н. Кладиев, А.С. Глазырин, Е.В. Боловин, В.И. Полищук // Известия Томского политехнического университета. Инжиниринг георесурсов. - 2019. - Т. 330. - № 4. - С. 158-177.

18. Steinwolf A. Random vibration testing beyond PSD limitation // Sound and Vibration - 2006. - V. 40. - P. 12-21.

19. Tandon N., Nakra B.C. Vibration and Noise Engineering. - New Delhi: I.K. International Publishing House Pvt. Ltd, 2017. $204 \mathrm{p}$.

20. Investigation on random vibration of a drillstring / H. Qiu, J. Yang, S. Butt, J. Zhong // Journal of Sound and Vibration. 2017. - V. 406. - P. 74-88.

21. Zhao W., Zhang Yi-Min. Reliability analysis of random vibration transmission path systems / Mechanical Systems and Signal Processing. - 2018. - V. 113. - P. 77-89.

22. Старовойтов Э.И., Тарлаковский Д.В. Теория упругости и пластичности. - М.: Физматлит, 2002. - 416 с.

23. Westergaard H.M. Theory of elasticity and plasticity. - Cambridge: Harvard University Press, 1952. - $176 \mathrm{p}$.

24. Pollock D. Physical Properties of Materials for Engineers. V. 3. Boca Raton: Press CRC, 2018. - 310 c.

\section{Информация об авторах}

Нижегородов А.И., доктор технических наук, профессор кафедры строительных, дорожных машин и гидравлических систем Иркутского национального исследовательского технического университета.

Гаврилин А.Н., кандидат технических наук, доцент отделения материаловедения Инженерной школы новых производственных технологий Национального исследовательского Томского политехнического университета.

Мойзес Б.Б., кандидат технических наук, доцент отделения контроля и диагностики Инженерной школы неразрушающего контроля и безопасности Национального исследовательского Томского политехнического университета.

Кладиев C.H., кандидат технических наук, доцент отделения электроэнергетики и электротехники Инженерной школы энергетики Национального исследовательского Томского политехнического университета.

Хамитов P.H., доктор технических наук, профессор кафедры электрической техники Омского государственного технического университета; профессор кафедры электроэнергетики Тюменского индустриального университета.
25. Тимошенко С.П., Донован Х.Я., Янг У.У. Колебания в инженерном деле. - М.: Машиностроение, 1985. - 472 с.

Weaver W. Jr., Tymoshenko S.P., Young D.H. Vibration Pro-
blems in Engineering. - New-York: Wiley-Interscience, 1967. $444 \mathrm{p}$.

Abingdon:

Нижегородов А.И. Анализ и синтез универсальной вибрационной машины с гидрообъемным возбуждением колебаний. Ч. 2 // Вестник машиностроения. - 2017. - № 12. - С. 3-9.

ская модульно-спусковая печь для обжига вермикулитовых концентратов // Строительные и дорожные машины. - 2015. № 9. - C. 12-17.

Нижегородов А.И. Экспериментальное определение коэффитериалов // Строительные материалы. - 2016 - № 11. C. $63-67$.

Поступила 26.08.2019 2. 


\title{
RESEARCH OF A NONLINEAR MODEL OF AN ELECTRIC FURNACE MOBILE BASE PLATE FOR FIRING BULK MINERALS
}

\author{
Anatoly I. Nizhegorodov', \\ nastromo_irkutsk@mail.ru
}

Alexey N. Gavrilin²,

tom-gawra@@list.ru

Boris B. Moyzes²,

mbb@tpu.ru

\section{Sergey N. Kladiev ${ }^{2}$, \\ kladiev@tpu.ru}

\author{
Rustam N. Khamitov ${ }^{3,4}$ \\ apple_27@mail.ru \\ ${ }^{1}$ Irkutsk National Research Technical University, \\ 83, Lermontov Avenue, Irkutsk, 664074, Russia. \\ 2 National Research Tomsk Polytechnic University, \\ 30, Lenin Avenue, Tomsk, 634050, Russia. \\ ${ }^{3}$ Omsk State Technical University, \\ 11, Mira Avenue, Omsk, 644050, Russia. \\ ${ }^{4}$ Tyumen Industrial University, \\ 38, Volodarsky street, Tyumen, 625000, Russia.
}

Relevance. Electric furnaces with a mobile base plate have drawbacks in the design of vibrotransport system. It is the platform located at an angle to the horizontal plane. It is relevant to use a horizontal base plate, which excludes spontaneous rolling of material rounded particles and eliminates the possibility of temperature traction occurrence inside the firing space, resulting in cooling furnace heating system as well as fired bulk material.

The aim of the research is to study the dynamics of a mobile platform with a base plate and a one-sided elastic element, which provides a strongly presented nonlinear elastic characteristic, and bulk material vibrotransportation over the surface of a horizontal base plate.

The object of the research is a mobile base plate of an electric furnace for bulk minerals firing.

The methods are based on the development of the design and model of a mobile base plate of an electric furnace for firing bulk minerals. The results of researching the properties of a special elastic element in the furnace mobile base plate allowed obtaining a nonlinear analytical model of its motion, which is characterized by an inclined "skeletal» curve of its amplitude-frequency characteristic. The research of the dynamics of an oscillatory system with a one-sided elastic element, providing strong non-linearity, confirmed that the system performs non-symmetrical vibrations with peak vibration acceleration values of 8,32 and $4,79 \mathrm{~m} / \mathrm{s}^{2}$ corresponding to the extreme positions of the base plate and differing in 1,74 times. It is shown that when the furnace is installed horizontally, the system sensitivity to fluctuations of excitation frequency, spring stiffness, friction forces and other factors, which change due to the influence of high temperature and external factors, is almost three times reduced. The effect of stable vibrotransportation of bulk material on the horizontal surface of the base plate in the thermal field of the furnace is confirmed.

\section{Key words:}

Electric furnace with a mobile base plate, dynamic system, nonlinear elastic element, "skeletal» curve, amplitude-frequency characteristic, dynamic system sensitivity, vibro-transportation, non-symmetrical vibrations.

\section{REFERENCES}

1. Balima F., Laurence Reinert L., An-Ngoc N., Le Floch S. Effect of the temperature on the structural and textural properties of a compressed K-vermiculite. Chemical Engineering Science, 2015, vol. 134, pp. 555-562.

2. Chen L., Pingxiao Wu P., Chen M., Liu T. Chen L., Pingxiao Wu P., Chen M., Liu T. Preparation and characterization of the eco-friendly chitosan/vermiculite biocomposite with excellent removal capacity for cadmium and lead. Applied Clay Science, 2018, vol. 159, pp. 74-82.

3. Industrial minerals \& rocks: commodities, markets, and used. Ed. by J.E. Kogal. Littleton, Society for Mining, Metallurgy, and Exploration, Inc., 2006. 1529 p.
4. Karatas M., Benli A., Toprak H.A. Effect of incorporation of raw vermiculite as partial sand replacement on the properties of selfcompacting mortars at elevated temperature. Construction and Building Materials, 2019, vol. 221, pp. 163-176.

5. Kariya J., Ryu J., Kato Y. Development of thermal storage material using vermiculite and calcium hydroxide. Applied Thermal Engineering, 2016, vol. 94, pp. 186-192.

6. Ngayakamo B., Eugene Park S. Evaluation of kalalani vermiculite for production of high strength porcelain insulators. Science of Sintering, 2019, vol. 51, no 2, pp. 1-10.

7. Rashad A.M. Vermiculite as a construction material - a short guide for Civil Engineer. Construction and Building Materials, 2016, vol. 125 , pp. 53-62. 
8. Zhang Y.N. Development and thermochemical characterizations of vermiculite $\mathrm{SrBr}_{2}$ composite sorbents for low-temperature heat storage. Energy, 2016, vol. 115, pp. 120-128.

9. Nizhegorodov A.I., Bryanskikh T.B., Gavrilin A.N., Moyzes B.B., Gradoboev A.V. Vavilova G.V. Testing a new alternative electric furnace for vermiculite concentrates heat treatment. Bulletin of the Tomsk Polytechnic University. Geo Assets Engineering, 2018, vol. 329, no. 4, pp. 142-153. In Rus.

10. Slavova Y., Marinova M. On determining the energy efficiency of the electric oven. $16^{\text {th }}$ Conference on Electrical Machines, Drives and Power Systems, ELMA. Varna, Bulgaria, 2019. Article number 8771681 .

11. Bauman V.A., Bykhovskiy I.I. Vibratsionnye mashiny i protsessy $v$ stroitelstve [Vibration machines and processes in construction]. Moscow, Vysshaya shkola Publ., 1977. 255 p.

12. Ahirrao N.S., Bhosle S.P., Nehete D.V. Dynamics and Vibration Measurements in Engines. Procedia Manufacturing, 2018, vol. 20 , pp. 434-439.

13. Vibratsii v tekhnike: Spravochnik. T. 2. Kolebaniya nelineynykh mekhanicheskikh sistem [Vibrations in technology: Handbook. Vol. 2. Vibrations of nonlinear mechanical systems]. Ed. by I.I. Blekhman. Moscow, Mashinostroenie Publ., 1979. 351 p.

14. Den Gartog Dzh. P. Mekhanicheskie kolebaniya [Mechanical vibration]. Moscow, Fizmatgiz Publ., 1960. 455 p.

15. Deng N., Ostadan F. Random vibration theory based seismic site response analysis. $14^{\text {th }}$ World Conference on Earthquake Engineering. Beijing, 2008. Article number 04-02-0024.

16. Gavrilin A.N., Bolovin E.V., Glazyrin A.S., Kladiev S.N., Polishchuk V.I. Resonant oscillations with a limiting amplitude in a vibration electromagnetic activator. Bulletin of the Tomsk Polytechnic University. Geo Assets Engineering, 2019, vol. 330, no. 1, pp. 201-213. In Rus.

17. Gavrilin A.N., Kladiev S.N., Glazyrin A.S., Bolovin E.V., Polishchuk V.I. Identification of parameters of vibration electromagnetic activator mechanical system using limiting near resonance frequency. Bulletin of the Tomsk Polytechnic University. Geo Assets Engineering, 2019, vol. 330, no. 4, pp. 158-177. In Rus.
18. Steinwolf A. Random vibration testing beyond PSD limitation. Sound and Vibration, 2006, vol. 40, pp. 12-21.

19. Tandon N., Nakra B.C. Vibration and Noise Engineering. New Delhi, I.K. International Publishing House Pvt. Ltd., 2017. $204 \mathrm{p}$.

20. Qiu H., Yang J., Butt S., Zhong J. Investigation on random vibration of a drillstring. Journal of Sound and Vibration, 2017, vol. 406 , pp. 74-88.

21. Zhao W., Zhang Yi-Min. Reliability analysis of random vibration transmission path systems. Mechanical Systems and Signal Processing, 2018, vol. 113, pp. 77-89.

22. Starovoytov E.I. Tarlakovskiy D.V. Teoriya uprugosti $i$ plastichnosti [Theory of elasticity and plasticity]. Moscow, Fizmatlit Publ., 2002. $416 \mathrm{p}$.

23. Westergaard H.M. Theory of elasticity and plasticity. Cambridge, Harvard University Press, 1952.176 p.

24. Pollock D. Physical Properties of Materials for Engineers. Vol. 3. Boca Raton, Press CRC, 2018. 310 p.

25. Timoshenko S.P., Donovan Kh.Ya., Yang U.U. Kolebaniya $v$ inzhenernom dele [Fluctuations in engineering]. Moscow, Mashinostroenie Publ., 1985. 472 p.

26. Weaver W. Jr., Tymoshenko S.P., Young D.H. Vibration Problems in Engineering. New-York, Wiley-Interscience, 1990. $624 \mathrm{p}$.

27. Halit E. Acceleration, Vibration, and Shock Measurement. Abingdon, Taylor \& Francis Group, CRC Press LLC, 2000.950 p.

28. Nizhegorodov A.I. Analysis and synthesis of a universal vibration machine with hydro-volume excitation of oscillations. P. 2. Vestnik mashinostroeniya, 2017, vol. 12, pp. 3-9. In Rus.

29. Nizhegorodov A.I. Energy- and resource-saving electric modulelaunching furnace for firing vermiculite concentrates. Stroitelnye i dorozhnye mashiny, 2015, vol. 9, pp. 12-17. In Rus.

30. Nizhegorodov A.I. Experimental determination of friction coefficients of some potentially thermoactive materials. Stroitelnye materialy, 2016, vol. 11, pp. 63-67. In Rus.

Received: 26 August 2019.

\section{Information about the authors}

Anatoly I. Nizhegorodov, Dr. Sc., professor, Irkutsk National Research Technical University.

Alexey N. Gavrilin, Cand. Sc., associate professor, National Research Tomsk Polytechnic University.

Boris B. Moyzes, Cand. Sc., associate professor, National Research Tomsk Polytechnic University.

Sergey N. Kladiev, Cand. Sc., associate professor, National Research Tomsk Polytechnic University.

Rustam N. Khamitov, Dr. Sc., professor, Omsk State Technical University; professor, Tyumen Industrial University. 